Electronic Supplementary Information (ESI)

\title{
Highly Hydrophobic Thermally Stable Liquid Crystalline Cellulosic
}

\section{Nanomaterials}

Eldho Abraham ${ }^{\mathrm{a}}$, Yuval Nevo ${ }^{\mathrm{a}}$, Rikard Slattegard ${ }^{\mathrm{b}}$, Noam Attias $^{a}$, Sigal Sharon ${ }^{\mathrm{a}}$, Shaul Lapidot $^{\mathrm{b}}$ and Oded Shoseyov ${ }^{\mathrm{a} \dagger}$

${ }^{\mathrm{a}}$ R.H. Smith Institute of Plant Sciences and Genetics and The Harvey M. Krueger

Family Center for Nanoscience and Nanotechnology, The Hebrew University of Jerusalem, Israel.

†email: shoseyov@agri.huji.ac.il,Fax: +972-8-9462283; Tel: +972-8-9489084

${ }^{b}$ Melodea Ltd, Faculty of Agriculture, Hebrew University of Jerusalem, Israel

Total number of pages: 5

Total number of figures: 4

Total number of tables: 0 


\section{Elemental analysis}

Elemental analysis of dry $\mathrm{CNC}$ and $\mathrm{Bu}-\mathrm{CNC}$ demonstrated a decreased weight percentage of elemental carbon from $47.77 \%$ in $\mathrm{CNCs}$ to $36.56 \%$ in $\mathrm{Bu}-\mathrm{CNC}$, caused by the increase in oxygen content from 42.79 to 54.87 upon butyric esterification (Figure S1). The degree of ester substitution was in line with the theoretical DS value of 2.1 and normalized ${ }^{13} \mathrm{C}$ NMR results discussed in the main manuscript. Elemental analysis of $\mathrm{CNC}$ and $\mathrm{Bu}-\mathrm{CNC}$ was performed with and without prior soxhlet purification of the samples. Both results strongly supported the theoretical DS value of 2.1 (same as from ${ }^{13} \mathrm{C} \mathrm{NMR}$ ) in Bu-CNC, irrespective of the soxhlet purification. The sulphur content of $\mathrm{CNC}(0.661 \%)$ determined from the elemental analysis corroborated with the sulphate ester content estimation $(0.34 \mathrm{mmol}$ sulfate half-ester per $1 \mathrm{~g}$ of cellulose). The sulfur content dropped to $0.03 \%$ following ester modification, which is reasonable for a DS of 2.1.

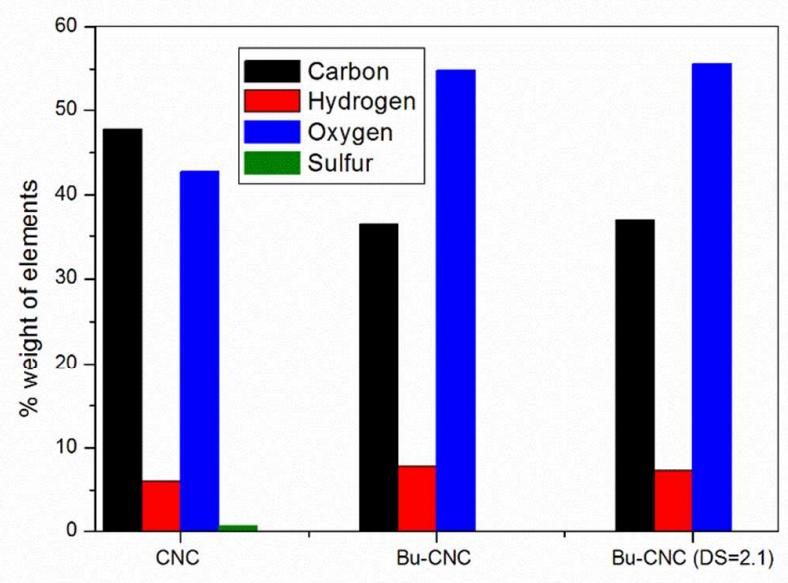

Figure S1. Elemental weight compositions

\section{XRD peak separation}

XRD patterns of $\mathrm{CNC}$ and $\mathrm{Bu}-\mathrm{CNC}$ were fitted with pseudo-voigt peak functions of Lorentzian components and the specific peaks of cellulose I and II were evaluated by peak separation method. The $\mathrm{CNC}$ and $\mathrm{Bu}-\mathrm{CNC}$ possessed multiple peaks of cellulose I and cellulose II, respectively (Fig. S2). CNC displayed characteristic peaks of cellulose I at $14.98^{\circ}, 22.37^{\circ}$ and $34.1^{\circ}$ (Fig. S2a). Bu-CNC had multiple peaks at 
$12.1^{\circ}, 16.2^{\circ}$ and $19.2^{\circ}$ (Fig. S2b), which correspond to cellulose II allomorphic crystallographic planes.
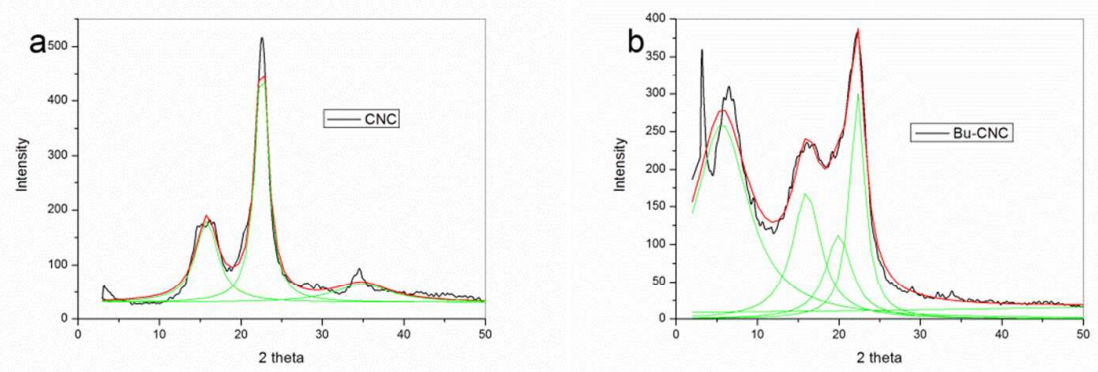

Figure S2. Peak separation of cellulose I in CNC (a) and cellulose II in Bu-CNC (b)

\section{$\mathrm{UV}$-vis spectrum of $\mathrm{CNC}$ and $\mathrm{Bu}-\mathrm{CNC}$}

$\mathrm{UV}$-Vis spectroscopy is an efficient means of investigating molecular interactions between cellulosic molecules, as manifested by material transparency. A comparison of the UV-Vis spectra of $\mathrm{CNC}$ and $\mathrm{Bu}-\mathrm{CNC}$ in dry film and ethanol suspension (2 wt.\%) showed clear differences in absorption (Figure S3). No absorption was recorded in the visible area $(400-700 \mathrm{~nm})$ for dry $\mathrm{CNC}$ and $\mathrm{Bu}-\mathrm{CNC}$ films and for $\mathrm{Bu}-$ $\mathrm{CNC}$ dispersed in ethanol. $\mathrm{CNC}$ in ethanol was opaque and absorbed in the visible area. The spectrum of Bu-CNC films displayed a band at $226 \mathrm{~nm}$, which is a bathochromic shift $(\Delta \lambda=20 \mathrm{~nm})$ from the CNC spectrum, owing to the ester aggregates in Bu-CNC molecules. It should be noted that there is indeed some contribution from oligomeric assemblies in addition to ester aggregates, ${ }^{1}$ which was quite obvious with ethanol dispersions of $\mathrm{CNC}$ and $\mathrm{Bu}-\mathrm{CNC}$ and is due to their polarity versus that of the dispersing medium. The spectrum of $\mathrm{CNC}$ in ethanol exhibited remarkably greater red-shifted absorption peaks when compared to $\mathrm{Bu}-\mathrm{CNC}$ in ethanol, reflective of the aggregation of cellulose crystals of CNC. 


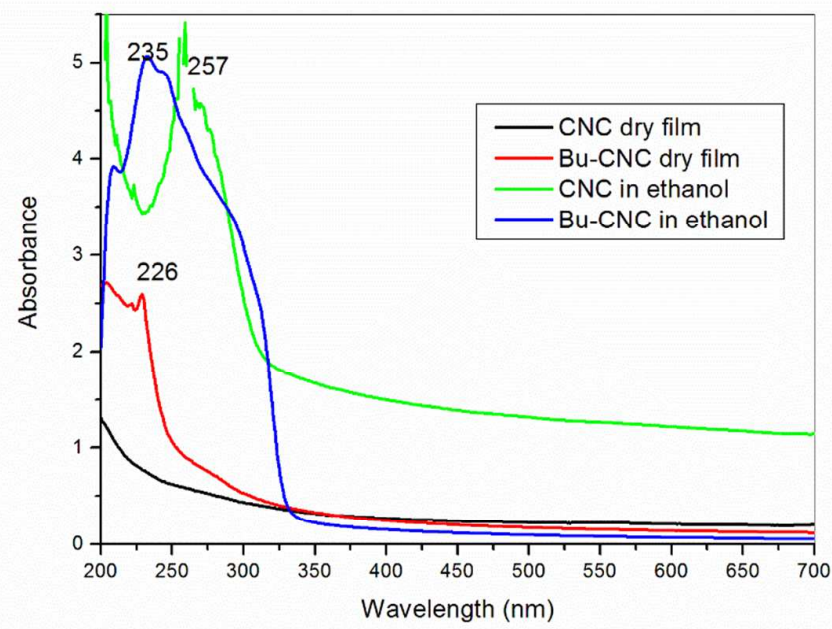

Figure S3. UV-vis spectrum of $\mathrm{CNC}$ and $\mathrm{Bu}-\mathrm{CNC}$

\section{Scanning electron microscopic analysis of the films}

SEM analysis was performed to characterize the surface morphology and layering patterns of the $\mathrm{CNC}$ and $\mathrm{Bu}-\mathrm{CNC}$ dried films (Fig. S4). A comparison between the SEM images of the cross-section of CNC (Fig. S4a-c) and Bu-CNC (Fig. S4d-f) films, at different magnifications, is shown. The cross-sections of the films provided some interesting insights which correlated with their layering pattern. In the case of CNC, the cellulosic layers were arranged in close proximity to one another (Fig. S4a-c), yielding a chiral nematic crystalline arrangement. In contrast, the greater interlayer spacing in Bu-CNC films (Fig. S4d-f), with an average distance of $70 \mathrm{~nm}$, suggested another degree of order in the molecular arrangement of Bu-CNC crystals. The bulky butyric group replacing the hydroxyls of $\mathrm{CNC}$ pulled apart $\mathrm{Bu}-\mathrm{CNC}$ layers and the decreased degree of intermolecular hydrogen bonding further contributed to crystal layer orientation with a smectic order of arrangement. ${ }^{2,3}$ The difference in arrangements of cellulosic layers due to the bulky ester pendants in $\mathrm{Bu}-\mathrm{CNC}$ is also responsible for its enhanced thermal stability. 

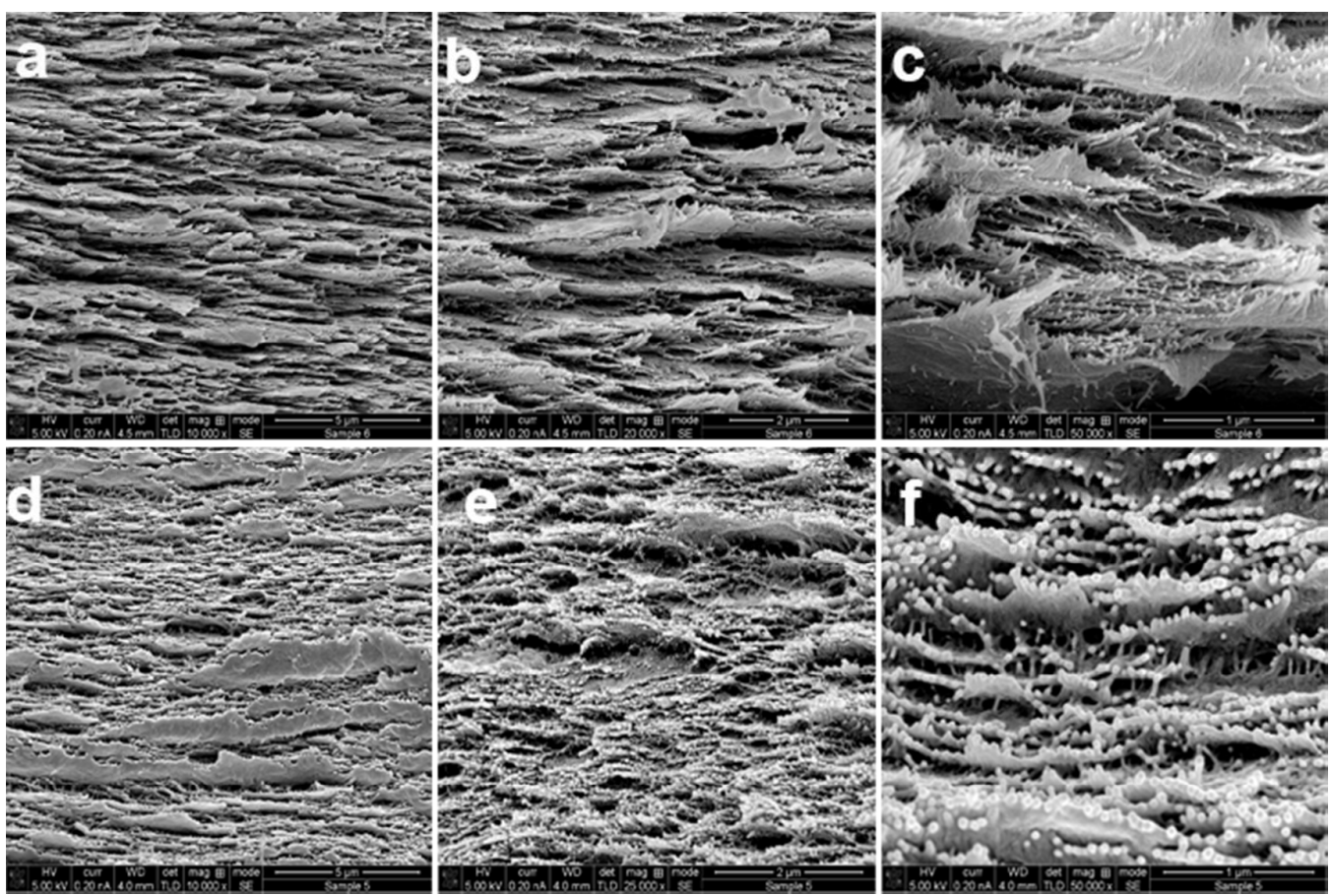

Figure S4. SEM of the cross-section of CNC (a-c) and Bu-CNC (d-f) films, at different magnifications

References

(1) Sakakibara, K.; Granström, M.; Kilpeläinen, I.; Helaja, J.; Heinilehto, S.; Inoue, R.; Kanaya, T.; Hill, J.P.; Nakatsubo, F.; Tsujii Y.; Ariga, K. LightHarvesting Nanorods Based on Pheophorbide-Appending Cellulose. Biomacromolecules 2013, 14, 3223-3230.

(2) Wang, N.; Ding, E.; Cheng, R. Preparation and Liquid Crystalline Properties of Spherical Cellulose Nanocrystals. Langmuir 2008, 24, 5-8.

(3) Cheung, C.C.Y.; Giese, M.; Kelly, J.A.; Hamad, W.Y.; MacLachlan, M.J. Iridescent Chiral Nematic Cellulose Nanocrystal/Polymer Composites Assembled in Organic Solvents. ACS Macro Letters 2013, 2, 1016-1020. 